\section{Infection Control Campaign at Evacuation Centers in Miyagi Prefecture after the Great East Japan Earthquake}

To the Editor-Japan is now experiencing a crisis as a result of the Great East Japan Earthquake (magnitude, 9.0) that occurred on March 11, 2011. ${ }^{1}$ The most devastated area was Miyagi prefecture in the Tohoku region, especially the coastal area, which experienced a great deal of damage as a result of the tsunami. The Fukushima nuclear power plant is dispersing radioactive substances as a result of the seismic damage to the plant. $^{2}$ As of April 1,2011, the death toll associated with the earthquake and the subsequent tsunami exceeded 6,700 , with more than 7,000 individuals missing in Miyagi prefecture. ${ }^{3}$ Approximately 71,000 people are living at 550 evacuation shelters. Many survivors are obliged to reside in the shelters under harsh and unsanitary conditions. Health care is likely to be insufficient for evacuees because there are few healthcare workers, including medical doctors, registered nurses, and public health nurses. There is a public health concern about the increased risk of infectious diseases, including acute respiratory infections, influenza, tuberculosis, and measles, under crowded living conditions and about diarrheal diseases and waterborne diseases that are typically seen after natural disasters. ${ }^{4,5}$ In Japan, an increase in the morbidity rate associated with pneumonia was reported after the 1995 Hanshin-Awaji earthquake. ${ }^{6}$ Therefore, the Tohoku Regional Infection Control Network has begun infection control activities to support evacuation centers in their fight against infectious diseases.

People who have lost their homes are crowded into each evacuation center (Figure 1). In most cases, there is no housing available for evacuees to live in, and the distance between families is less than $1 \mathrm{~m}$, which suggests the difficulty of conducting droplet precautions. Influenza was epidemic from February through March in Japan, ${ }^{7}$ and continuous monitoring of influenza at evacuation centers is needed. We found that some individuals could not wear masks properly, even if the mask supply were sufficient to provide masks for all residents. Room ventilation in evacuation centers also tends to be poor, because it remains cold in Miyagi prefecture until late spring. If small rooms in which to isolate patients with influenza-like illnesses are unavailable in evacuation centers, partitioning family units by using corrugated cardboard may be acceptable.

Running water is unavailable or insufficient as a result of damage to the water supply system. Hand hygiene depends on the use of alcohol-based hand sanitizers, despite the limited supply of such sanitizers. In addition, many residents have little understanding of hand hygiene because they are not healthcare professionals. Poor compliance with hand hy- giene increases the risk of cross infection, particularly when cooking and eating, using temporary lavatories, and processing garbage and infectious waste. Education regarding the importance of hand hygiene before food preparation and after using toilets is needed, and alcohol-based hand sanitizers need to be installed in the most visible spots.

Many evacuation centers still experience unsanitary conditions as a result of poor environmental maintenance. Floors and toilets remain dirty unless no outdoor shoes are allowed and regular cleaning is done. Inappropriate disposal of infectious waste, including vomitus, feces, and diapers, can lead to the transmission of infectious pathogens. It is essential to disseminate basic knowledge and skills for cleaning and disinfecting the shelter environment.

To achieve effective infection control measures in Miyagi prefecture, we require the cooperation and contribution of many hospitals in the region. The Tohoku Regional Infection Control Network was established in 1999 to fight against infectious diseases and to promote infection control activities. ${ }^{8}$ The operation center is located in the infection control unit of Tohoku University Hospital and is connected with more than 100 regional healthcare facilities. The network works functionally and collaboratively on infection control activities at evacuation centers and hospitals in disasterstricken areas, including activities such as (1) infectious diseases consultation, (2) infection control educational programs and training, (3) infection control interventions, and (4) regional cooperation with local government against infectious diseases. The network enables us to rapidly respond to infection control issues in efficient ways, especially in terms of information dissemination and resource allocation. Thus, the network has an important role in solving infection control issues and improving infection control practices in the Tohoku region. We will continue our infection control activities at evacuation centers after the most disastrous earthquake on record.

\section{ACKNOWLEDGMENTS}

We thank all participants in the Tohoku Regional Infection Control Network for their cooperation and contributions.

Potential conflicts of interest. All authors report no conflicts of interest relevant to this article.

\author{
Hajime Kanamori, $\mathrm{MD}, \mathrm{PhD}$; $^{1,2}$ \\ Hiroyuki Kunishima, $\mathrm{MD}, \mathrm{PhD}{ }^{1}$ \\ Koichi Tokuda, MD, PhD, MPH; \\ Mitsuo Kaku, MD, $\mathrm{PhD}^{1}$
}

Affiliations: 1. Department of Infection Control and Laboratory Diagnostics, Internal Medicine, Tohoku University Graduate School of Medicine, 

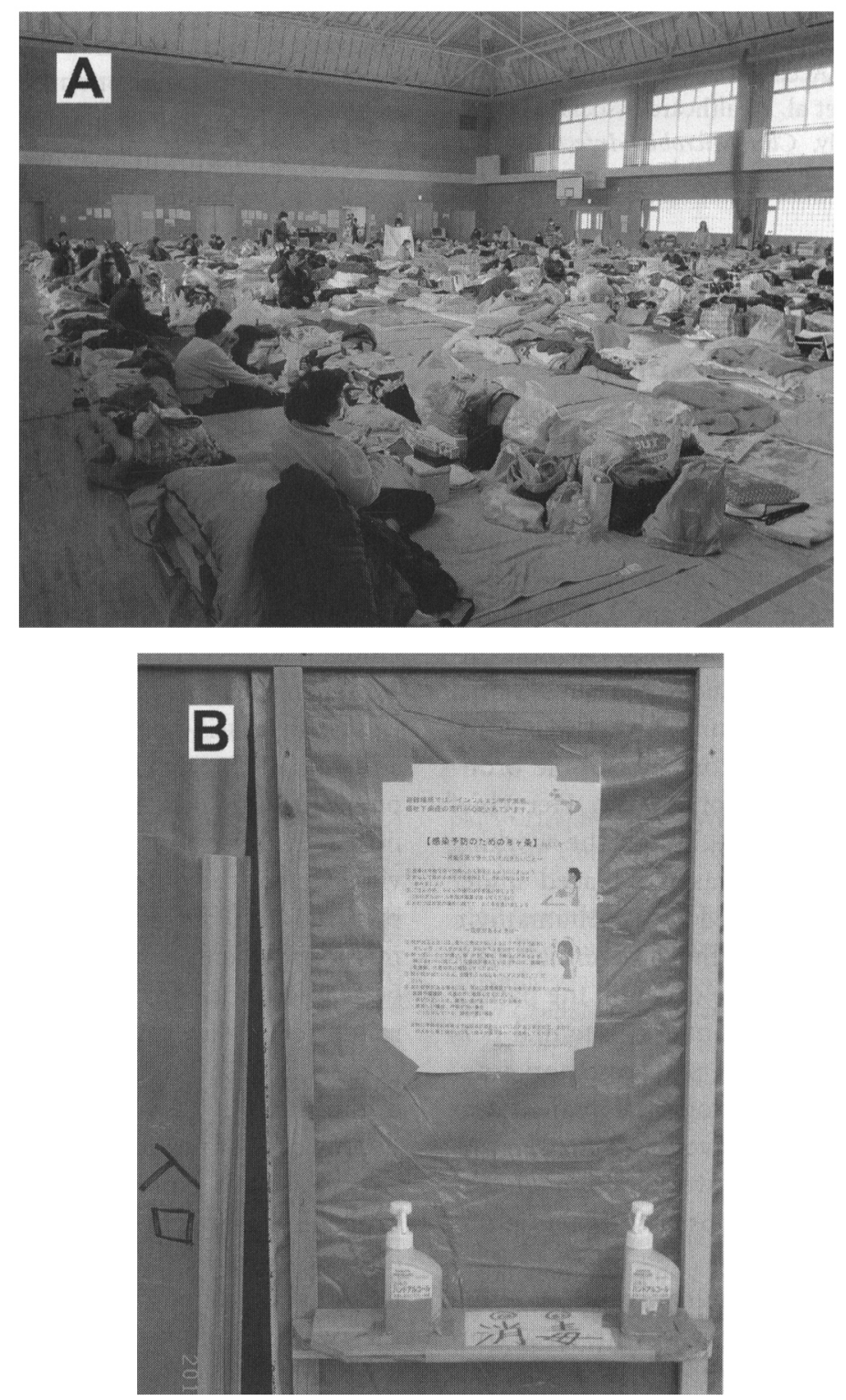

FIGURE 1. Crowded living conditions in an evacuation center $(A)$ and an infection control poster and alcohol-based hand sanitizers put on a temporary toilet $(B)$.

Sendai, Japan; 2. Miyagi Cardiovascular and Respiratory Center, Kurihara, Japan.

Address correspondence to Hajime Kanamori, $\mathrm{MD}, \mathrm{PhD}$, Department of Infection Control and Laboratory Diagnostics, Internal Medicine, Tohoku University Graduate School of Medicine, 1-1 Seiryo-machi, Aoba-ku, Sendai 980-8574, Japan (kanamori@med.tohoku.ac.jp).

Infect Control Hosp Epidemiol 2011;32(8):824-826

(C) 2011 by The Society for Healthcare Epidemiology of America. All rights reserved. 0899-823X/2011/3208-0016\$15.00. DOI: $10.1086 / 661224$

\section{REFERENCES}

1. Prime Minister of Japan and His Cabinet. Countermeasures for the Great East Japan Earthquake. http://www.kantei.go.jp/foreign/ incident/index.html. Accessed April 5, 2011.
2. Nuclear and Industrial Safety Agency. Countermeasures for Tohoku-Pacific Ocean earthquake. http://www.nisa.meti.go.jp/ english/index.html. Accessed April 1, 2011.

3. Miyagi Prefectural Government. Information on earthquake damage [in Japanese]. http://www.pref.miyagi.jp/pdf/4010900.pdf. Accessed April 1, 2011.

4. Watson JT, Gayer M, Connolly MA. Epidemics after natural disasters. Emerg Infect Dis 2007;13:1-5.

5. World Health Organization. Flooding and communicable diseases fact sheet. Wkly Epidemiol Rec 2005;3:21-28.

6. Matsuoka T, Yoshioka T, Oda J, et al. The impact of a catastrophic earthquake on morbidity rates for various illnesses. Public Health 2000;114:249-253.

7. Infectious Disease Surveillance Center, National Institute of In- 
fectious Diseases. Infectious agents surveillance report: flash report of influenza virus in Japan, 2010/11 season. http:// idsc.nih.go.jp/iasr/influ-e.html. Accessed April 5, 2011.

8. Marcel JP, Alfa M, Baquero F, et al. Healthcare-associated infections: think globally, act locally. Clin Microbiol Infect 2008;14: 895-907.

\section{Infection Control Implications of Nosocomial Pertussis Exposure in a Hospitalized Adult during Influenza Season}

To the Editor-During influenza season, some disorders present as influenza-like illnesses (ILIs) and may mimic influenza (eg, human parainfluenza virus 3 infection and Legionnaires' disease).$^{1-3}$ At our hospital during this influenza season, influenza in hospitalized adults has been due to human seasonal influenza $A(\mathrm{H} 3 \mathrm{~N} 2)$ and swine influenza $A(\mathrm{H} 1 \mathrm{~N} 1)$ virus. Recently, an elderly woman with a history of chronic bronchitis was admitted to our hospital for an ILI. Her chief complaint was persistent dry cough. Although the working diagnosis was influenza, the patient was not placed under influenza (droplet) precautions or treated with oseltamanivir. Although pertussis was considered in the differential diagnosis and pertussis diagnostic tests were ordered, the patient was not placed under droplet precautions for potential pertussis. When polymerase chain reaction performed on a nasopharyngeal swab sample obtained from the patient was reported to have results that were positive for Bordetella pertussis on hospital day 3,51 healthcare workers and the patient's roommate had already been exposed to potential infection.

A 71-year-old woman presented to our institution with a nonproductive cough. Her illness had begun with nasal congestion approximately 1 week before presentation. The patient then developed a nonproductive cough with some shortness of breath. She denied fever, chills, nausea, vomiting, diarrhea, or myalgias. She reported having recent contact with her grandchildren, who had upper respiratory tract infections. Her medical history was remarkable for chronic bronchitis, diabetes mellitus, systemic lupus erythematosus, heart failure, and osteoarthritis. She had quit smoking 11 years earlier.

At admission to the hospital, the patient was afebrile with a pulse of 92 beats $/ \mathrm{min}$, blood pressure of $122 / 45 \mathrm{~mm} \mathrm{Hg}$, and a respiratory rate of 15 breaths/min. She was in no distress but was constantly coughing. Her physical examination had findings that were within normal limits except for bilateral rhonchi with wheezing and mild lower-extremity edema. Her initial white blood cell count was 7,500 cells $/ \mathrm{mm}^{3}$ (69\% neutrophils, $19 \%$ lymphocytes, $9 \%$ monocytes, and $2 \%$ eosinophils), and her platelet count was 184,000 cells $/ \mathrm{mm}^{3}$. Her serum transaminase and ferritin levels were normal, but her erythrocyte sedimentation rate of $82 \mathrm{~mm} / \mathrm{h}$ (normal range, $1-35 \mathrm{~mm} / \mathrm{h}$ ) was highly elevated. Chest radiographs obtained at admission had normal findings. To treat possible community-acquired pneumonia, the patient initiated a regimen of ceftriaxone ( $1 \mathrm{~g}$ administered intravenously every 24 hours), azithromycin ( $500 \mathrm{mg}$ administered intravenously every 24 hours), and methylprednisolone. Her rapid influenza test result was negative, and her nasopharyngeal respiratory viral fluorescent antibody panel had results that were negative for influenza A and B virus, parainfluenza viruses, respiratory syncytial virus, adenovirus, and metapneumovirus. Her Chlamydophila pneumoniae and Mycobacterium pneumoniae immunoglobulin (Ig) M titers were not elevated. B. pertussis IgM titer was highly elevated at $2.3 \mathrm{U} / \mathrm{mL}$ (normal range, $0.0-0.9 \mathrm{U} / \mathrm{mL}$ ), and her $B$. pertussis IgG titer was $4 \mathrm{U} / \mathrm{mL}$ (normal range, $0-9 \mathrm{U} / \mathrm{mL}$ ).

Pertussis, which is caused by $B$. pertussis, is most common in childhood but is not uncommon among elderly adults. Unlike classic pertussis in children, adult pertussis presents as a persistent nonproductive cough that lasts for several weeks. Pertussis pneumonia, although less common among adults than among children, may also occur. ${ }^{4-8}$ In adults hospitalized with ILIs, pertussis may go unnoticed or undiagnosed. Because pertussis is highly contagious and is spread via droplets, the potential for nosocomial spread among hospitalized patients with pertussis is great. ${ }^{9,10}$ At hospital admission, patients with suspected or known pertussis should be placed under droplet precautions and treated with a macrolide for 5 days. After macrolide therapy, coughing often persists, but the contagious potential of pertussis should be diminished.

This case of pertussis in a hospitalized adult during influenza season is emblematic of a common infection control problem. Although her physicians suspected pertussis, they did not place the patient under droplet precautions until the nasopharyngeal pertussis polymerase chain reaction result for the swab sample was reported 3 days after the hospital admission. During this 72 -hour period, there were 51 healthcare workers with potential exposure ( 8 of whom had close contact exposures). The patient's roommate was also exposed to pertussis for 3 days and was given azithromycin prophylaxis.

This case underscores important epidemiologic and infection control lessons. First, although every ILI in a hospitalized adult during influenza season is not due to influenza virus, adults who are admitted to the hospital with ILIs should be placed under influenza (droplet) precautions at hospital admission pending diagnostic testing for influenza. Second, if pertussis, which is a highly contagious infectious disease, is in the differential diagnosis, the patient should be under appropriate droplet precautions pending test results to decrease the potential for nosocomial pertussis. Although influenza was the working diagnosis in this case, there would have been no nosocomial pertussis exposures because droplet precautions are appropriate for pertussis as well as for influenza. The clinical presentation of adult pertussis may mimic an ILI or influenza with dry cough, leukocytosis, and no infiltrates 